\title{
EchoGéo
}

$14 \mid 2010$

Afrique, 50 ans d'indépendance : Dynamiques spatiales, identités, circulations

\section{Les investisseurs étrangers à l'assaut des terres agricoles africaines.}

Réflexions sur le dernier avatar des politiques agricoles post coloniales

\section{Florence Brondeau}

\section{(2) OpenEdition}

\section{Journals}

\section{Édition électronique}

URL : https://journals.openedition.org/echogeo/12008

DOI : $10.4000 /$ echogeo.12008

ISSN : 1963-1197

\section{Éditeur}

Pôle de recherche pour l'organisation et la diffusion de l'information géographique (CNRS UMR 8586)

\section{Référence électronique}

Florence Brondeau, «Les investisseurs étrangers à l'assaut des terres agricoles africaines. », EchoGéo [En ligne], 14 | 2010, mis en ligne le 13 décembre 2010, consulté le 31 juillet 2021. URL : http:// journals.openedition.org/echogeo/12008; DOI : https://doi.org/10.4000/echogeo.12008

Ce document a été généré automatiquement le 31 juillet 2021.

EchoGéo est mis à disposition selon les termes de la licence Creative Commons Attribution - Pas d'Utilisation Commerciale - Pas de Modification 4.0 International (CC BY-NC-ND) 


\section{Les investisseurs étrangers à l'assaut des terres agricoles africaines.}

Réflexions sur le dernier avatar des politiques agricoles post coloniales

\section{Florence Brondeau}

\section{Introduction}

1 La ruée des investissements privés vers les terres agricoles des pays du Sud a été particulièrement médiatisée ces derniers mois. Ce processus est qualifié d'« accaparement des terres » ou d' «agro-colonialisme " par les ONG et bon nombre d'organisations paysannes (Grain, 2008), de façon plus neutre d'« acquisition » de terres agricoles par la Banque Mondiale, $d$ '« appropriation à grande échelle» ou encore d'« investissements directs étrangers (IDE) »... Ces termes révèlent des interprétations différentes et souvent partielles. Les ONG dénoncent la spoliation des terres et la violation des droits des populations locales alors que l'IIED ${ }^{1}$ pose le débat en ces termes : "Land grab or development opportunity ?"(Cotula, 2009).

2 Les appropriations foncières des États ou des sociétés étrangères ne constituent certes pas une nouveauté : elles jalonnent l'histoire récente de l'Afrique. L'émoi suscité par le processus actuel doit être replacé dans un contexte où le spectre des récentes émeutes de la faim (2008) plane toujours sur les villes du Sud. En outre, l'accès à la terre reste un sujet sensible, particulièrement en Afrique, où les exploitants familiaux, pilier de la production agricole, aspirent à une sécurisation foncière passant par une reconnaissance juridique des droits coutumiers (Chauveau, 2006).

3 Peu d'informations scientifiques sont toutefois disponibles pour dresser une analyse objective de cette évolution aussi rapide que récente. Les sources restent essentiellement journalistiques et les rapports réalisés par les ONG présentent des analyses fortement engagées et sans doute pas toujours objectives. La source qui fait 
référence aujourd'hui est un rapport récent demandé par la FAO et l'IFAD et réalisé par l'IIED (Cotula, 2009).

Dans ce contexte, cet article veut avant tout exposer quelques clés d'analyse qui permettent de envisager ce processus dans une dimension spécifique à l'Afrique.

\section{Les terres agricoles africaines au cœur d'un réseau de transactions aux règles opaques}

L'accélération des investissements directs étrangers dans le domaine foncier est un phénomène mondial dont l'enjeu principal réside dans la maîtrise de surfaces agricoles. La carte 1 a été réalisée à partir de données croisées issues de différentes sources bibliographiques (L'Hebdo, 2009 n 36 ; Cotula, 2009; Grain, 2008 et 2009). Elle n'a pas vocation à représenter de façon exhaustive des flux liés aux IDE: les données disponibles étant très incomplètes et les accords négociés ou envisagés se révélant innombrables et parfois rapidement caducs. Cette carte permet toutefois de visualiser la place de l'Afrique dans cet imbroglio de flux et de transactions.

Carte 1 - Les investisseurs étrangers à l'assaut des terres agricoles : un phénomène planétaire encore mal évalué

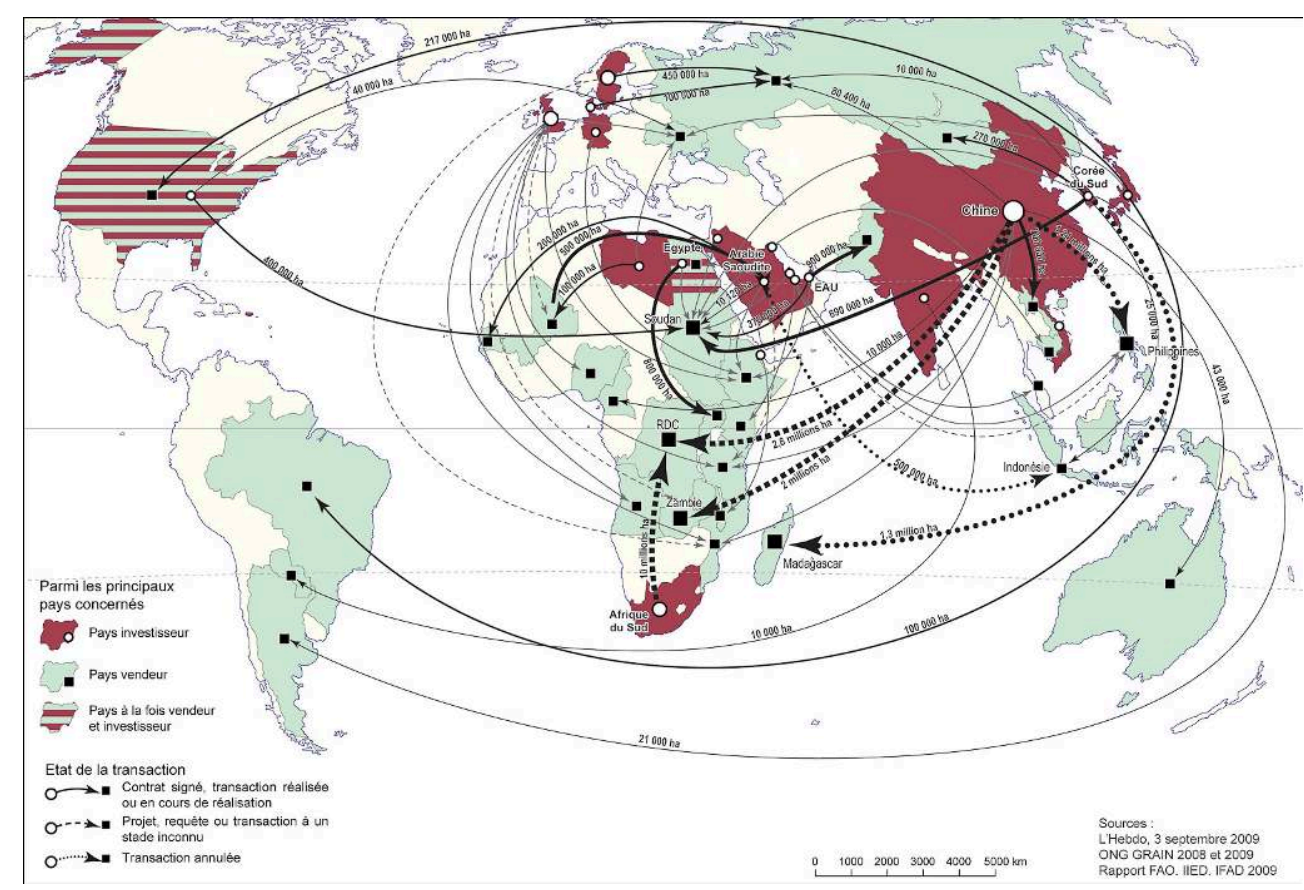

Sources : d'après l'Hebdo 3 septembre 2009 ; Grain 2008 et 2009 ; rapport FAO-IIED-IFAD 2009.

6 Au-delà de sa rapidité, c'est aussi la diversité et la complexité des partenariats qui caractérisent l'évolution en cours, tandis que de nouveaux enjeux semblent se profiler dans un contexte marqué par la mondialisation des flux financiers, les menaces de pénurie de produits alimentaires et surtout par la faillite des politiques de développement post-coloniales.

7 La diversité des accords pressentis forme un kaléidoscope impossible à reconstituer précisément, du fait de l'imprécision et de la rareté des données. Rares sont les travaux de recherche réalisés sur la base d'études de terrain solides. Les sources comme les 
chiffres avancés diffèrent; les analyses sont souvent ponctuelles et basées sur des témoignages et des interviews. Le rapport réalisé par l'IIED à Madagascar, en Ethiopie, au Soudan, au Ghana et au Mali, repose sur un inventaire des contrats de concession foncière portant sur des surfaces supérieures à 1000 ha. Les transactions répertoriées concerneraient 2,5 millions d'hectares depuis 2004; beaucoup n'ont toutefois pas dépassé le seuil des négociations, certains sont en attente, d'autres ont été annulées ("affaires » Daewoo et Varun à Madagascar), la plupart restent encore à l'état de projet. Ces accords ne sont pas passés d'État à État mais entre les gouvernements des pays d'accueil et des sociétés d'investissement souvent mixtes. La société Malibya Agriculture en constitue un exemple: les capitaux de la société Laïco y sont très largement majoritaires et sa création a précédé la mise en place du projet. Dans le cas des investissements avortés de Daewoo à Madagascar, c'est la filiale Madagascar Futur Entreprise qui avait entrepris les négociations (Beuret, 2009). Il faut en outre préciser que l'«achat» de terres agricoles ne constitue pas la règle. Les États africains ne vendent pas leurs terres au sens strict du terme mais attribuent des baux emphytéotiques d'une durée de 18 à 99 ans.

8 Les enjeux qui suscitent une telle ruée des investissements étrangers vers les terres agricoles africaines sont divers. D'abord, la politique d'externalisation de la production agricole consiste, pour un pays, à développer une production agricole à l'extérieur de son territoire. Le renchérissement brutal des prix des produits alimentaires de base pendant l'année 2008 justifie, en partie, la politique d'investissement pratiquée par les États dépendant de leurs importations ou qui sont susceptibles de le devenir. Ainsi, bien que dans des contextes économiques et démographiques très différents, l'Arabie Saoudite, les Emirats Arabes Unis, le Qatar, mais aussi l'Egypte, la Chine, l'Inde, la Corée du Sud ou le Japon ont lancé une véritable offensive sur les terres agricoles à l'étranger. En outre, la production de d'agrocarburantsse développe sur de très vastes surfaces pour cultiver des palmiers à huile, de la canne à sucre ou du Jatropha curcas. Par ailleurs certains investissements reposent sur de pures spéculations financières exercées par des sociétés d'investissement en quête de placements rapidement lucratifs.

Ces transactions sont également associées à des enjeux géopolitiques qui dépassent le secteur agricole. Les pays du Golfe, la Libye et l'Arabie Saoudite tentent depuis longtemps de tisser des liens politiques et économiques privilégiés avec les pays africains musulmans. Dans le cadre de la Cen-Sad ${ }^{2}$, la Libye s'est vue attribuer 100000 ha de terres potentiellement irrigables dans la région du Macina au Mali, au prétexte de sécuriser l'approvisionnement alimentaire de l'ensemble de la Cen-Sad et de promouvoir le développement économique des états membres. L'Arabie Saoudite a une stratégie très ciblée qui s'associe à celle des pays du Golfe. Ainsi, l'Organisation de la Conférence Islamique soutient un certain nombre de projets, financés partiellement par la Banque Islamique de Développement et par la Private Islamic Development Corporation. La société Foras Investment Company serait financée par des capitaux privés saoudiens, par l'Arabie Saoudite, et par la BID³. Ses prospections porteraient sur l'aménagement de 700000 ha au Mali, au Sénégal et en Mauritanie. L'Union Economique et Monétaire Ouest-Africaine (UEMOA)a désigné le Mali comme le grenier à riz potentiel de l'Afrique de l'Ouest. Dans ce contexte, l'Office du Niger est destiné à devenir un pôle de production macro-régional. Officiellement, 100000 ha y ont été accordés au Sénégal pour renforcer sa production rizicole. L'UEMOA a lancé une étude portant sur 11000 ha; 5500 ha devraient être aménagés avant 2012 (Dave, 2008). 
10 Il n'est pas toujours possible de préciser la nature des contrats de concession et des accords passés entre les différents protagonistes. Le rapport FAO-IIED-IFAD avance certes des chiffres précis : en Ethiopie, 603000 ha ont été attribués à des investisseurs étrangers pour 78,5 millions de dollars; au Mali, 162850 ha ont été cédés pour 292 millions de dollars... Il faut toutefois spécifier que ces sommes correspondent pour l'essentiel à des promesses d'investissements sous la forme de grands travaux de génie civil: construction de routes, de ports, de canaux d'irrigation, de logements, d'hôpitaux... Le Qatar tente ainsi de négocier avec le gouvernement kényan l'attribution de 40000 ha dans le delta de la Tana en échange du financement d'un port moderne dans lî̂le voisine de Lamu. La Libye finance au Mali la construction d'un grand canal et de routes goudronnées dans la zone office du Niger, tandis que Malibya a achevé la construction de la cité ministérielle de Bamako et d'une mosquée à Ségou. Le projet Foras comprendrait la construction d'une Chambre de Commerce et d'Industrie à Dakar et la réalisation de 60000 logements sociaux au Mali... La création d'emplois constitue la contrepartie la plus fréquente : 10000 emplois doivent accompagner la mise en place du projet Malibya. Ces compensations peuvent également porter sur des transferts de technologie et de savoir-faire. Ainsi, les sociétés chinoises présentes dans la basse vallée du Sénégal pour produire du sésame s'engagent à former les paysans à la riziculture, à l'utilisation des engrais et des semences hybrides.

11 Ces partenariats, quels qu'ils soient, sont formalisés par des contrats de quelques pages, souvent confidentiels, qui ne comprennent pas de clauses précises sur des points essentiels (Cotula, 2009). Les engagements fiscaux de ces sociétés d'investissement ne sont jamais mentionnés, sauf quand elles ont réussi à négocier des exonérations. La direction de Malibya Agriculture a ainsi obtenu une exonération des droits de douane et de la redevance qui porte sur l'eau à usage agricole dans la zone Office du Niger. La part de la production agricole injectée dans les circuits commerciaux internes n'est jamais spécifiée. Les déplacements de populations et les indemnisations ne sont pas non plus envisagés, ou de façon trèssommaire. Ces imprécisions suscitent évidemment de vives inquiétudes dans les milieux associatifs paysans.

\section{La faisabilité et la durabilité des projets en question : analyse des dérives pressenties}

12 Les arguments utilisés par les investisseurs comme par les pays d'accueil pour justifier ces projets méritent d'être confrontés à l'analyse. Les investissements engagés sont destinés à accroître rapidement la production alimentaire sur le territoire national et donc à promouvoir un processus de croissance agricole. Initieront-ils pour autant un quelconque processus de développement?

13 A une échelle macro-économique, il n'est pas certain que les recettes soient significatives : beaucoup de sociétés disposent d'exonérations fiscales. A l'échelle locale, les retombées économiques restent très incertaines. D'après les visites réalisées sur le terrain par une délégation de la $\mathrm{CNOP}^{4}$ sur le site du projet Malibya, les collectivités locales ne reçoivent aucune taxe sur l'extraction des carrières utilisées pour la construction de la route. Ailleurs, les promesses d'emploi offrent peu d'opportunités de promotion sociale pour des paysans devenus salariés : chez Elfora, en Ethiopie, les salariés gagnent officiellement l'équivalent de 40 dollars par mois et souvent beaucoup moins (Zaugg, 2009). Ces emplois sont par ailleurs précaires et saisonniers. La main- 
d'œuvre est utilisée pour la réalisation des grands travaux de génie civil, la construction des canaux d'irrigation et des routes, pour le montage des serres ou pour les récoltes. En outre, la plupart de ces grandes exploitations sont mécanisées et ne procurent finalement des emplois qu'en nombre limité. Les contrats restent d'ailleurs très flous sur le sujet : Malibya s'engage à procurer 10000 emplois sans spécifier ni leur nature, ni les niveaux de rémunération. Les emplois qualifiés échappent par ailleurs au marché local : la société saoudienne Elfora a confié un de ses projets d'horticulture à un ingénieur néerlandais. Les travaux de génie civil sont réalisés par des sociétés qui amènent pour certaines leur propre main-d'œuvre, à l'image de la CGC (China GeoEngineering Corporation) en charge des travaux engagés par Malibya.

Par ailleurs, il faut craindre qu'en l'absence d'études de faisabilité sérieuses préalables, ces projets ne s'exposent à des déboires inévitables... Ainsi en dépit de la rusticité tant vantée de Jatropha curcas, les plantations implantées dans le Sud-ouest de Madagascar ne tiennent d'ores et déjà pas toutes leurs promesses (Moreau, 2010). Le projet de canne à sucre mené depuis le début des années 2000 par la société sud africaine SOSUMAR ${ }^{5}$ à Markala (zone Office du Niger) a bien du mal à démarrer : des 14000 ha prévus à terme, seuls quelques dizaines d'hectares sont exploités. Dans cette même région, seuls 5,5\% des surfaces attribuées à l'UEMOA sont en cours d'aménagement alors que les travaux d'aménagement menés par Malibya sont confrontés à des retards imputables aux revendications des populations locales, qui bloquent régulièrement depuis quelques mois le travail des équipes de la société chinoise CGC.

En outre, on peut difficilement admettre que ces projets soient à même d'assurer la sécurisation alimentaire des populations alors que la plus grande partie de la production est destinée à approvisionner les marchés extérieurs. Faute d'accord spécifique, leur contribution à l'approvisionnement du marché national est donc susceptible d'être fort aléatoire en fonction des besoins des pays investisseurs et des prix pratiqués sur le marché mondial. En ce sens, la spéculation sur les produits alimentaires risque de compromettre la stabilité des prix et la régularité des approvisionnements attendus par la population. En outre, des millions d'hectares sont d'ores et déjà voués à la production d'agrocarburants. Par ailleurs, une croissance agricole basée sur l'utilisation de technologies de pointe importées accroît la dépendance de ces États vis-à-vis de l'extérieur. Les semences hybrides, et de surcroît les semences OGM, ne sont pas reproductibles et sont assujetties à une utilisation massive d'intrants. Ainsi, les paysans qui, dans le cadre des accords de transfert de technologie et de formation seront convertis à cette forme de riziculture intensive, devront s'approvisionner en semences et en engrais, soit sur le marché international soit par l'intermédiaire des usines de production semencières et phytosanitaires implantées par des sociétés étrangères. Ceci risque de compromettre la viabilité de telles expériences, qui s'inscriront alors dans la longue histoire de précarité économique de l'agriculture irriguée de l'Afrique de l'Ouest. Ainsi, une usine chinoise de production de semences hybride sera construite à Boky Wéré dans le cadre du projet Malibya. Par ailleurs, on craint que la multiplication de ces projets ne donne lieu à des expérimentations hasardeuses en matière d'OGM. La COPAGEN (Coalition pour la Protection du Patrimoine Génétique Africain) s'inquiète de voir la zone Office du Niger se transformer en "une forêt d'expérimentation sans aucune transparence sur les types de semences qui sont introduits» (Grain, 2009). Le Parlement malien a voté en décembre 2008 
une loi extrêmement controversée en matière de sécurité sur les biotechnologies (Cnop-Infos, 2008).

L'impact socio-économique et environnemental déjà perceptible dans certaines régions suscite de vives inquiétudes dans les milieux paysans et associatifs. Les recompositions foncières majeures inhérentes à ces projets d'agro-business remettent en question toute sécurité quant à l'accès à la terre et aux ressources associées. Les titres de propriété en bonne et due forme sont rares dans ces régions, où le droit coutumier constituait la norme reconnue par les populations. Certains de ces titres datent de la période coloniale ; beaucoup ne sont plus valables ou sont contestés et ne protègent pas les paysans d'expropriations éventuelles. La plupart des pays concernés ont légiféré de manière à sécuriser l'accès au foncier des grands projets. Les sociétés investissant en Ethiopie bénéficient de baux de 30 à 99 ans sur des surfaces de plusieurs dizaines de milliers d'hectares pour des sommes de l'ordre de 10 à 12 dollars par hectare et par an (Zaugg, 2009). Ce sont parfois les meilleures terres qui font l'objet de ces attributions, en particulier les terres potentiellement irrigables même si elles étaient jusqu'à présent faiblement peuplées et mises en valeur, comme certaines basses terres éthiopiennes. En ce sens le "Land grabbing" se double d'un "Water grabbing». Ainsi, la société Fri-El Green Power (Italie) a prévu de détourner une partir de la rivière Omo, dont dépend toute une région en Ethiopie. Au Mali, les surfaces allouées à Malibya se situent en tête de réseau d'irrigation de l'office du Niger et les prélèvements, dont le volume n'a pas été évalué, menacent de priver d'eau les parcelles situées en aval du projet (carte 2). La Libye a d'ailleurs anticipé la question du partage de l'eau en obtenant du gouvernement malien la priorité de la desserte en saison sèche. Ce projet poursuit des travaux d'aménagement depuis octobre 2008, sans que ni la répartition de l'eau entre les différents utilisateurs, ni la concurrence potentielle entre la production énergétique et l'irrigation n'aient été envisagées, tandis que l'impact des prélèvements en eau n'a pas été calculé sur le régime du delta intérieur du Niger en aval. 
Carte 2 - Les projets bénéficiant d'attributions foncières à grande échelle dans la région de Macina (Mali, Office du Niger)

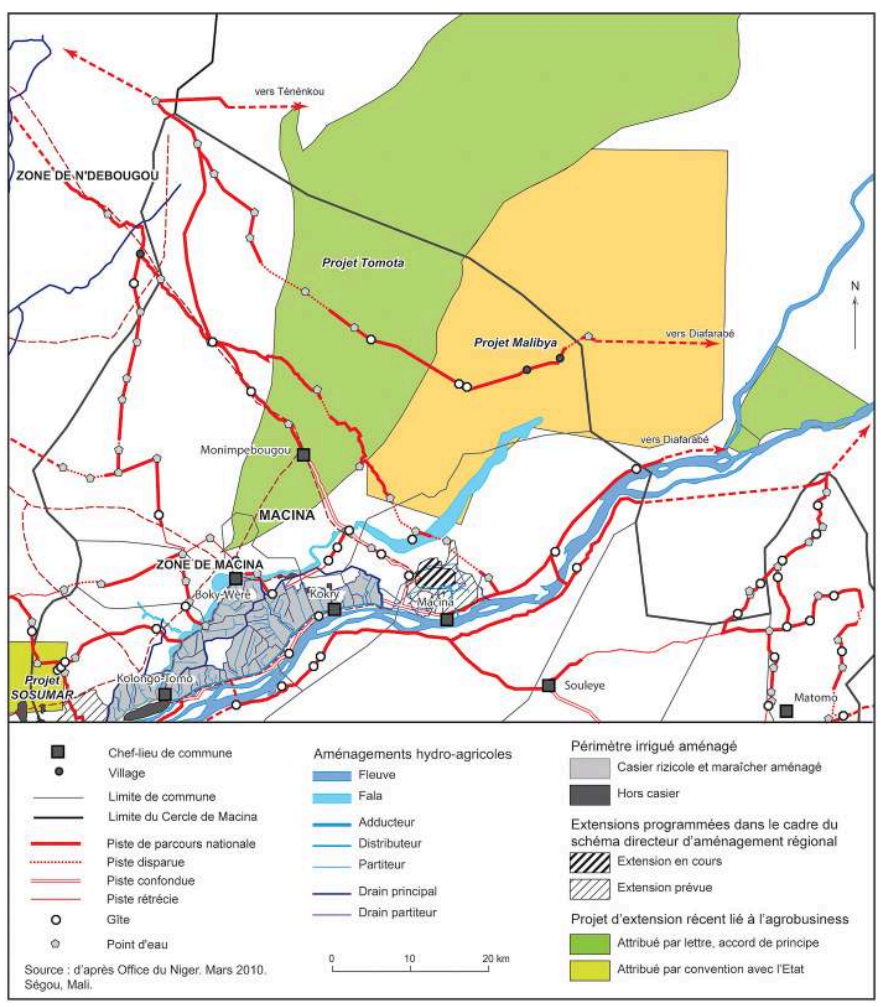

Source : d'après Office du Niger, mars 2010

Certes, les techniques d'irrigation mises en œuvre dans le cadre de ces projets d'agrobusiness permettent de limiter la consommation en eau: certains systèmes d'irrigation calculent automatiquement les quantités d'eau et de fertilisants nécessaires et les distribuent à chaque plant par un système de goutte à goutte (projet Elfora dans la région d'Awassa en Ethiopie, projet Grands moulins du Sénégal dans la basse vallée du Sénégal). En outre, certaines cultures sont pratiquées sous serre, ce qui présente l'avantage de limiter les pertes par évaporation. Mais le gigantisme des aménagements programmés laisse augurer une explosion des consommations en eau qui n'est, la plupart du temps, pas évaluée, et qui suscite des inquiétudes quant à la durabilité et au coût environnemental de ces projets (illustrations 1 à 3 ). Se pose en outre la question de la pollution susceptible d'accompagner les techniques de production intensives assujetties à l'utilisation massive d'intrants. 
Illustration 1 - Pancarte annonçant le démarrage des travaux zone Macina

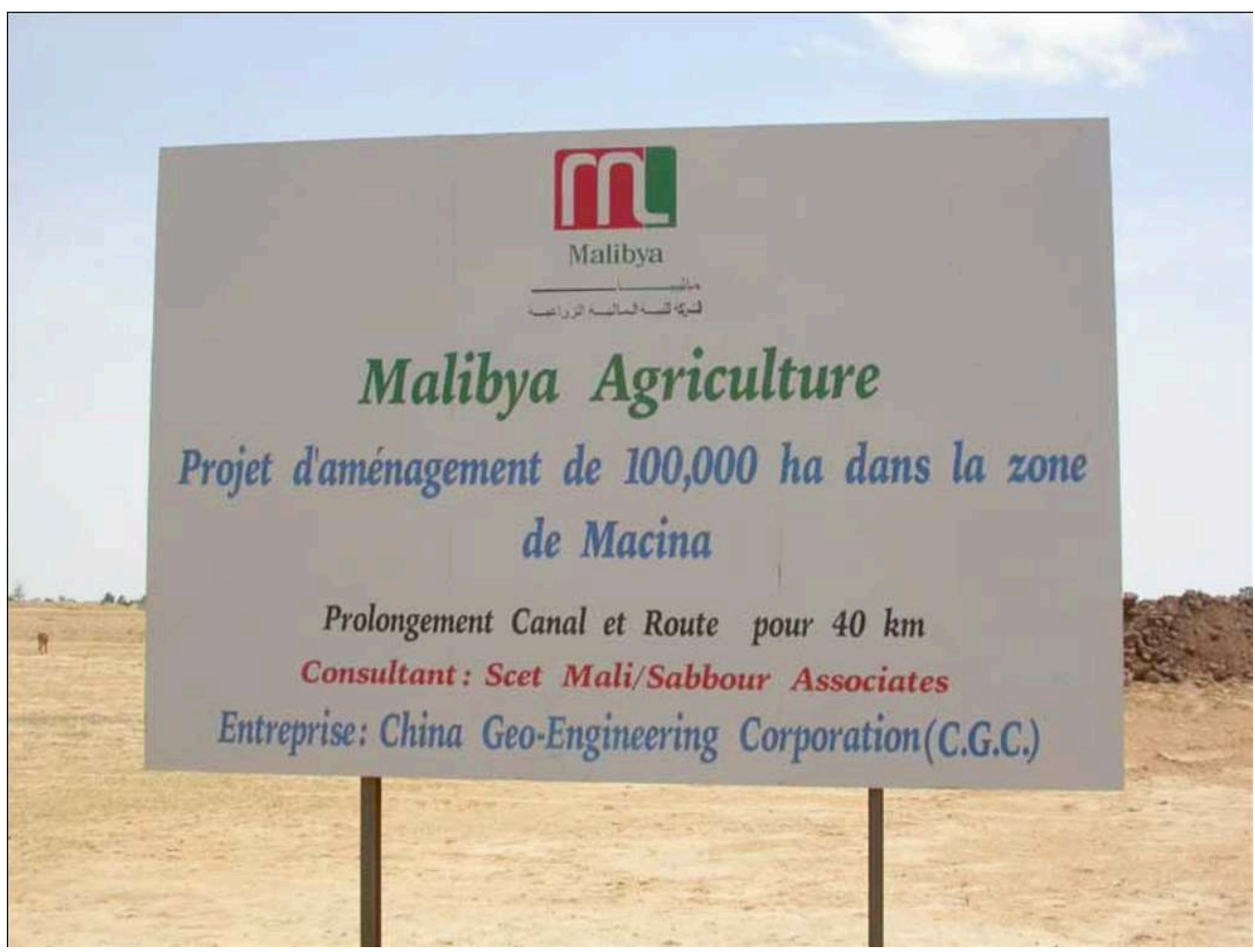

Auteur : Florence Brondeau, février 2010.

Illustration 2 - Travaux d'élargissement du canal de Boky Véré du projet Malibya ; tronçon Tolongotomo - Boky Véré

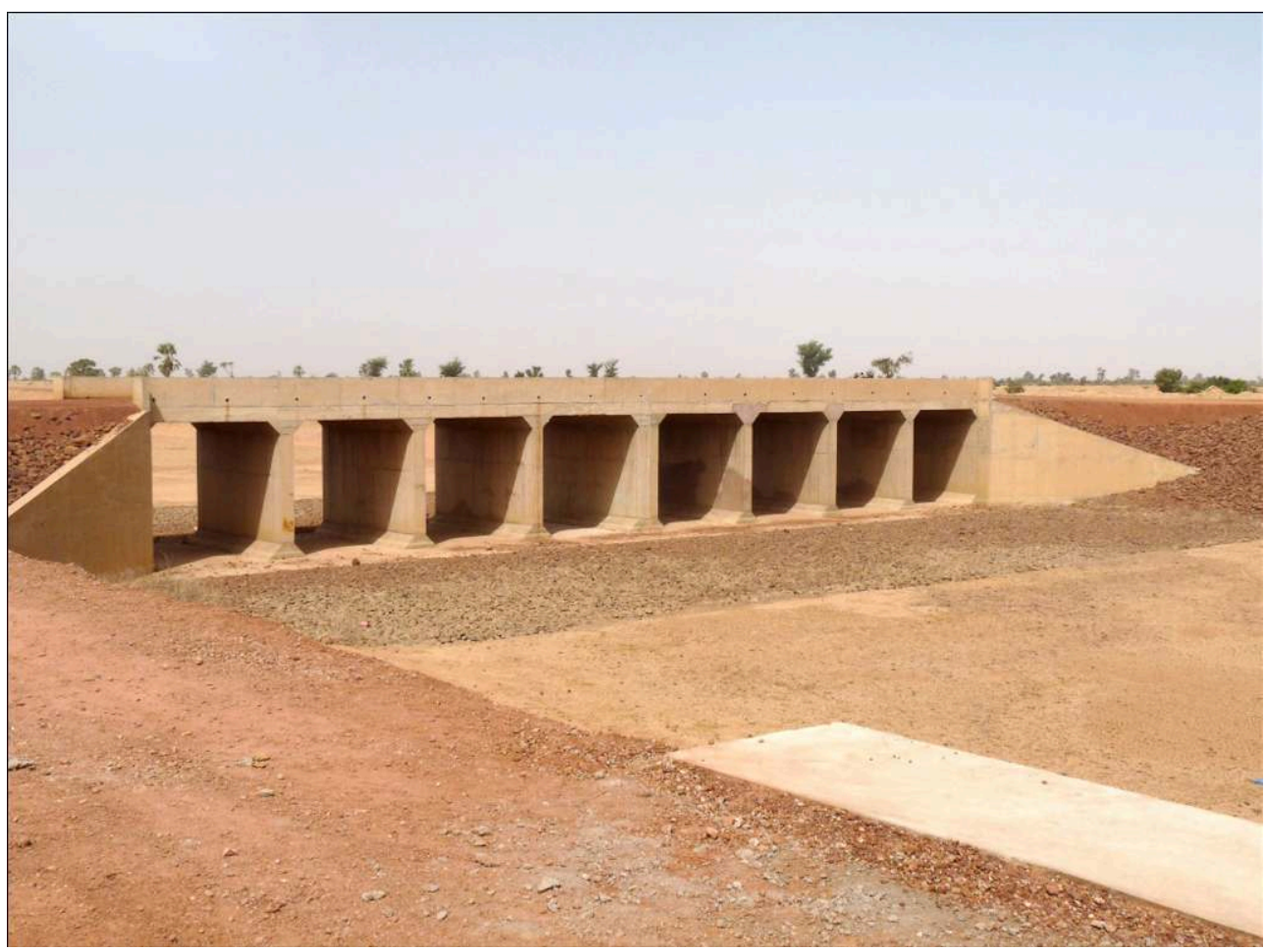

Auteur : Florence Brondeau, février 2010. 


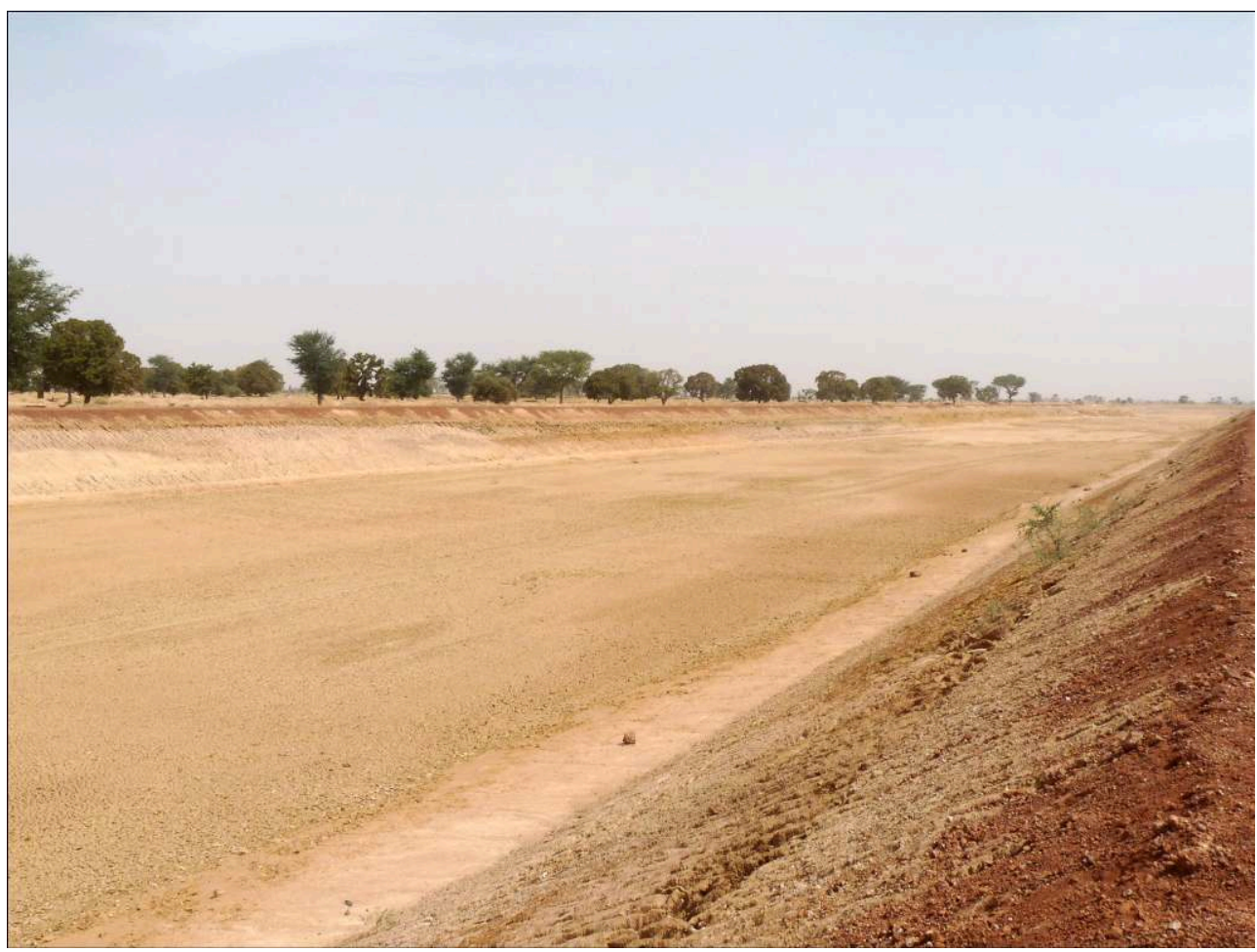

Auteur : Florence Brondeau, février 2010

Dans les zones aménagées, les conditions de dédommagement, les opérations de déplacement des populations et des réattributions foncières sont très inégales selon les projets et parfois non définies. Ainsi, en Ethiopie, les programmes saoudiens menés par Elfora ne prévoient pas d'indemnisation pour la terre elle-même. Les paysans spoliés reçoivent tout juste l'équivalent de 10 années de récoltes. Les éleveurs quant à eux ne perçoivent rien. Le projet Malibya ne prévoit quant à lui aucune clause précise au sujet des populations déplacées : le problème est délégué aux collectivités locales. En juillet 2009, les expropriations concernaient 150 familles ; 58 d'entre elles devaient bénéficier de dédommagements (Kone, 2009). Le PACT (Programme d'Appui aux Collectivités Territoriales), chargé de la mise en place des Conventions de gestion des ressources dans le Macina, aurait dû logiquement constituer un partenaire privilégié ; or, les responsables n'ont pas été contactés. Cela tient au fait que les terres attribuées sont considérées comme vacantes quand elles ne font pas l'objet d'une mise en valeur permanente: jachères et zones de pâture sont donc assimilées à des terres non exploitées. Les grands organismes internationaux n'échappent pas à cette interprétation erronée des systèmes de production traditionnels, suivis en cela par les gouvernements des pays concernés. «Sur 74 millions de terres arables, seuls 14 à 18 millions sont exploités à ce jour... » (Ministre de l'agriculture éthiopien, Zaugg, 2009). De ce fait, certains projets doivent établir des clôtures et engager des milices privées pour assurer la protection des plantations quand l'armée ou la police nationale n'intervient pas directement. Des populations nomades afar qui venaient faire pâturer leurs troupeaux en saison sèche ont ainsi été expulsées par la société Elfora en Ethiopie et ont dû accepter une sédentarisation forcée sur des parcelles à vocation pastorale qu'elles ont dû acheter. (Zaugg, 2009). 
19 Sur le projet Malibya, les premières tensions commencent à apparaître : la construction du bâtiment du bureau de contrôle du projet a été réalisée sur une des pistes principales de déplacements des troupeaux, une autre est obstruée par la construction d'une route et d'un canal (carte 2).

20 Dans ce contexte, comment concilier cette tendance à la privatisation du foncier au profit des investisseurs avec les réformes foncières initiées dans le cadre de la décentralisation (Mali, Sénégal) ou de l'établissement des Plans Fonciers Ruraux ? Par ailleurs, la sécurisation revendiquée par les paysans ne passe pas forcément par la généralisation des immatriculations à titre individuel à l'échelle de la parcelle (Chauveau, 2006). Les populations agricoles restent attachées au maintien des formes collectives de gestion et au principe de plurifonctionnalité des ressources et des territoires.

21 La négociation de contrats équitables constitue un objectif essentiel des organisations paysannes et des ONG internationales qui se sont mobilisées ces derniers mois. Le ROPPA (Réseau des organisations paysannes et de producteurs de l'Afrique de l'Ouest) multiplie les protestations contre ce processus $d$ ' "accaparement des terres». Le sommet du G8 en juillet 2009, à l'instar de la FAO et des Nations Unies (de Schutter, 2008), a insisté sur une nécessaire réglementation de ces investissements fonciers.

\section{Conclusion}

Ce processus de prise de contrôle de terres agricoles africaines par des acteurs étrangers, encore mal connu et inachevé, suscite à ce stade d'analyse un certain nombre de questions. La précipitation avec laquelle les gouvernements acceptent ces contrats laisse tout d'abord perplexe. Elle doit nous interroger sur la réalité du choix dont ces autorités disposent. D'une part, ces pays sont confrontés à l'échec des politiques de développement agricole menées jusque là. D’expériences socialistes en reconversions au libéralisme, les gouvernements indépendants ont toujours sacrifié le secteur agricole et la paysannerie au profit de l'approvisionnement à bas coût des populations urbaines. Ne faisant pas preuve d'une plus grande cohérence, au gré des aléas géopolitiques, des priorités économiques et des paradigmes développementalistes successifs, les instances internationales n'ont pas non plus soutenu le secteur agricole, ou de façon trop marginale, alors qu'une politique d'importation des produits alimentaires de base à bas coût a été systématiquement privilégiée (Boris, 2010). Les paysanneries locales sont donc incapables à ce jour de produire des excédents suffisants pour investir et moderniser les exploitations, ainsi que répondre aux besoins croissants des populations urbaines. D'autre part, les gouvernements en place doivent, sous la pression des populations et de leurs partenaires financiers, assumer des programmes visant à sécuriser l'approvisionnement alimentaire; à l'image du GOANA (Grande Offensive pour la Nourriture et l'Abondance) au Sénégal, du Plan Initiative Riz au Mali, ou du MAP (Madagascar Action Plan). Or, leurs disponibilités financières ne leur permettant pas d'investissements de grande envergure, ces programmes encouragent les investissements étrangers. Dans ce contexte, les propositions des sociétés étrangères peuvent effectivement apparaître comme de véritables opportunités, à tel point que certains pays en sont demandeurs. L'Ethiopie dresse un inventaire des terres qui seront proposées aux investisseurs : 2,7 millions d'hectares auraient été délimitées à ce jour (Zaugg, 2009). La position du responsable de la FAO 
pour l'Afrique de l'Est, Mafa Chipeta, est révélatrice de la situation de bon nombre d'Etats africains: "Cela fait des années que nous prêchons pour que des investisseurs s'intéressent à l'agriculture de ce pays. Alors maintenant qu'ils viennent, on ne va pas les dissuader. [...] On ne nourrira jamais la population éthiopienne à la force des bras des petits paysans; seule l'agriculture intensive et les technologies importées le peuvent. [...](Zaugg, 2009).

L'ouverture de l'accès au foncier aux IDE semble bien une des conséquences directes des échecs des politiques de développement agricole précédentes. Elle s'inscrit dans une tendance nette à la remise en question du modèle de développement agricole basé sur les exploitations familiales au profit d'un modèle basé sur l'agrobusiness. Cela implique une politique d'attribution foncière conditionnée par les capacités d'investissements des exploitants, qu'ils soient nationaux ou étrangers. Les IDE ne constituent donc qu'un aspect de ce processus d'appropriation foncière à grande échelle.

\section{BIBLIOGRAPHIE}

Beuret M. 2009. Terres nourricières. Le grand défi alimentaire. L'Hebdo, dossier spécial Le grand défi alimentaire, $\mathrm{n}^{\circ} 36$, http://www.hebdo.ch

Beuret M., 2009. Madagascar. Le Dragon chassé de la Grande Ile. L'Hebdo, dossier spécial Le grand défi alimentaire, $\mathrm{n}^{\circ} 36$, http://www.hebdo.ch

Boris J.-P., 2010. Main basse sur le riz. Paris, Fayard, 220 p.

Brasserie V., Ouadrago M.G., 2008-2009. La quête des terres agricoles en Afrique subsaharienne. Grain de se,l déc. 2008-fév.2009, $\mathrm{n}^{\circ} 45$.

Chauveau J.-P. et al,. 2006. Modes d'accès à la terre, marchés fonciers, gouvernance et politiques foncières en Afrique de l'Ouest. IIED/CLAIMS, London.

Clavreul L. , 2009. Au Mali les nouvelles mises en culture bénéficient surtout aux investisseurs libyens. Le Monde, 16 avril 2009.

Cnop-Info. Loi sur la sécurité dans les biotechnologies. Bulletin d'Information de la Coordination Nationale des Organisations Paysannes au Mali. $\mathrm{N}^{\circ}$ Spécial, Novembre 2008 http://www.sosfaim.be/ $\mathrm{pdf} / \mathrm{fr} / \mathrm{ds} 86$

Colin J.-Ph., Lemeur Y., Leonard E. 2009. Les politiques d'enregistrement des droits fonciers. Du cadre légal aux pratiques locales. Paris, Karthala.

Comby J., 1998. La fabrication de la propriété. In Propriété et environnement. Paris, Dalloz.

Comby J., 2004. La propriété, de la Déclaration des droits au Code civil. Etudes foncières, n 108.

Cotula L. et a., 2009. Land grab or development opportunity? Agricultural investment and international land deals in Africa. IIED. FAO. IFAD, $120 \mathrm{p}$. 
Dave B., 2008. L'Office de tous les plans agricoles. In Le mali est-il une puissance agricole ?, Défis sud $n^{\circ} 86$.

Défis Sud, 2009. Dossier Terres accaparées, paysans exclus. № 89.

de SCHUTTER O. (entretien avec.) La faim n'est pas une fatalité, l'alimentation est un droit. SOS FAIM, déc. $2008, \mathrm{n}^{\circ} 86$.

Grain, 2008. Main basse sur les terres agricoles en pleine crise alimentaire et financière. Rapport, octobre 2008, http://www.grain.org/briefings/?id=212

GTZ, 2009.Foreign Direct Investments FDI in land in Mali. 34 p. http://www2.gtz.de/dokumente/ bib/gtz2010-0064en-foreign-direct-investment-mali.pdf

Koe A., 2009. Mali. Terres rizicoles de l'Office du Niger : grogne paysanne contre l'occupation libyenne. Le Républicain, 4 sept. 2009, Bamako.

Laville-Delville Ph., Durand-Lasserve A. 2008. Gouvernance foncière et sécurisation des droits fonciers dans les pays du Sud. Livre blanc des acteur français de la coopération. Travaux du Comité technique Foncier et Développement. Paris AFD-MAE-DgCiD.

Le Roy E., Karsenty A., Bertrand A., 1996. La sécurisation foncière en Afrique. Paris, Karthala, 379 p.

Merlet M., 2009. Les phénomènes d'appropriation à grande échelle des terres agricoles dans les pays du Sud et de l'Est. Etudes foncières, n 142, novembre-décembre 2009.

Moreau S., 2009. Conférence Développement de l'agro-business et recompositions foncières à Madagascar. 5 janvier 2009, Séminaire du Master GAELE. Université Paris 4 Sorbonne.

Zaugg J.. 2009. Ethiopie. L'heure de la moisson a sonné. L’Hebdo, dossier spécial Le grand défi alimentaire, $\mathrm{n}^{\circ} 36, \mathrm{http}: / /$ www.hebdo.ch

\section{NOTES}

1. IIED : International Institute for Environment and Development.

2. Communauté des Etats Sahélo-Sahariens.

3. BID : Banque Islamique de Développement.

4. CNOP : Coordination Nationale des Organisations Paysannes. Mali.

5. SOSUMAR : Société Sucrière de Markala.

\section{RÉSUMÉS}

Les terres agricoles africaines sont convoitées par de nombreux investisseurs étrangers, ce qui se matérialise par une accélération des transactions portant sur des concessions foncières à grande échelle. Ces investissements répondent à des enjeux très divers et font l'objet de contrats rudimentaires, dont les clauses exactes sont souvent méconnues. Ce phénomène très médiatisé est extrêmement controversé et suscite de virulentes critiques, alors que les analyses scientifiques sur le sujet sont encore très rares. Au regard des effets pervers pressentis et pour 
certains déjà perceptibles, il peut être perçu comme une des dérives d'un nouveau modèle de croissance agricole basé sur la promotion de l'agrobusiness.

The African farmlands are the object of the greed of numerous foreign investors which materializes by an acceleration of the transactions carrying on the concession of lands on a largescale. These investments correspond to very diverse stakes and give rise to rudimentary contract the exact clauses which are often underestimated. This very mediatized phenomenon is extremely debated and arouses virulent criticisms while the scientific analyses on the subject are still very rare. Towards the anticipated perverse effects and for some already perceptible, he can be perceived as one of the drift of a new agricultural growth model based on the promotion of the agrobusiness.

\section{INDEX}

Mots-clés : agrobusiness, droits fonciers, gestion durable des ressources, investissements étrangers

Keywords : agrobusiness, foreign investments, land laws, sustainable management of the resources

\section{AUTEUR}

\section{FLORENCE BRONDEAU}

Florence Brondeau est maître de conférences à l'université Paris 4-Sorbonne et membre de l'UMR 8185 EneC. florence.brondeau@paris-sorbonne.fr 